\title{
Effect of Helicobacter pylori eradication on gastric carcinogenesis
}

\author{
Judith Romero-Gallo ${ }^{1,2}$, Elizabeth J Harris ${ }^{3}$, Uma Krishna ${ }^{1,2}$, Mary Kay Washington ${ }^{3}$, Guillermo I Perez-Perez ${ }^{4,5}$ \\ and Richard M Peek Jr $r^{1,2,6}$
}

Chronic gastritis induced by Helicobacter pylori is the strongest known risk factor for gastric adenocarcinoma, yet the effects of bacterial eradication on carcinogenesis remain unclear. Animal models provide important insights into factors that are involved in gastric carcinogenesis, and we previously utilized such a model to demonstrate that an in vivoadapted $H$. pylori strain, 7.13 , rapidly and reproducibly induces inflammation-mediated gastric carcinoma. In the current study, we used this bacterial strain as a prototype to define the role of targeted antimicrobial therapy in gastric carcinogenesis. Mongolian gerbils were infected with $\mathrm{H}$. pylori for 4 or 8 weeks, treated with antimicrobial agents or vehicle, and then euthanized at 8 weeks after the completion of therapy. All infected gerbils developed gastritis; however, inflammation was significantly attenuated in animals receiving antimicrobial therapy. Gastric dysplasia or cancer developed in $>60 \%$ of the gerbils that remained persistently colonized with $\mathrm{H}$. pylori, but in none of the animals treated with antibiotics following 4 weeks of infection. Infection with $H$. pylori for 8 weeks prior to therapy resulted in an attenuation, but not complete prevention, of pre-malignant and malignant lesions. Similarly, antibiotic therapy initiated at 4 , but not 8 , weeks after $H$. pylori challenge significantly reduced expression of the Th1 pro-inflammatory cytokine interferon- $\gamma$ within colonized gastric mucosa. These results indicate that treatment of $H$. pylori in this model decreases the incidence and severity of lesions with carcinogenic potential. The effectiveness of eradication is dependent upon the timing of intervention, providing insights into mechanisms that may regulate the development of malignancies arising within the context of inflammatory states.

Laboratory Investigation (2008) 88, 328-336; doi:10.1038/labinvest.3700719; published online 7 January 2008

KEYWORDS: inflammation; cytokine; dysplasia; gastric cancer; antibiotics

Gastric adenocarcinoma is the second leading cause of cancer-related death in the world. ${ }^{1-6}$ Approximately 649000 persons die from this malignancy each year and 5-year survival rates in the United States are $<15 \% .^{2}$ Helicobacter pylori is a Gram-negative bacterial species that selectively colonizes gastric epithelium and induces chronic inflammation, a host response that increases the risk for cancer of the distal stomach. ${ }^{1,7-10}$ Several studies have demonstrated that inappropriate Th1-mediated T-cell responses towards $H$. pylori facilitate the development of gastric injury, and the majority of $H$. pylori antigen-specific T-cell clones isolated from infected gastric mucosa produce higher levels of the Th1 cytokine interferon- $\gamma($ IFN- $\gamma)$ than the Th2 cytokine IL- $4 .{ }^{11,12}$ Based upon these data, the World Health Organization has classified $H$. pylori as a class I carcinogen for gastric cancer, and as virtually all infected persons have superficial gastritis, it is likely that the organism plays a causative role early in the progression to adenocarcinoma. However, the effects of eliminating $H$. pylori on the development of gastric cancer remain unclear.

Two large randomized controlled trials to evaluate the effects of anti- $H$. pylori therapy on the incidence of gastric cancer in humans have been performed in China. ${ }^{13,14}$ In one study, no significant benefit was identified for $H$. pylori eradication for the prevention of incident cases of gastric cancer over a mean follow-up period of 7.5 years. However, treatment of $H$. pylori did significantly decrease gastric cancer risk in infected individuals without pre-malignant lesions. ${ }^{13}$ In the second trial, $H$. pylori treatment resulted in statistically significant reductions in the combined prevalence of gastric

\footnotetext{
'Division of Gastroenterology, Department of Medicine, Vanderbilt University School of Medicine, Nashville, TN, USA; ${ }^{2}$ Department of Cancer Biology, Vanderbilt University School of Medicine, Nashville, TN, USA; ${ }^{3}$ Department of Pathology, Vanderbilt University, Nashville, TN, USA; ${ }^{4}$ Department of Medicine, New York University, New York, NY, USA; ${ }^{5}$ Department of Microbiology, New York University, New York, NY, USA and ${ }^{6}$ Department of Veterans Affairs Medical Center, Nashville, TN, USA Correspondence: Dr RM Peek Jr, MD, Division of Gastroenterology, Vanderbilt University School of Medicine, 1030C MRB IV, 2215B Garland Avenue, Nashville, TN 37232-2279, USA. E-mail: richard.peek@vanderbilt.edu

Received 29 October 2007; revised 05 December 2007; accepted 05 December 2007
} 
atrophy, intestinal metaplasia, dysplasia, and cancer, but did not decrease the risk of gastric cancer when analyzed as a single entity. ${ }^{14}$ A study performed in Colombia reported that successful treatment of $H$. pylori decreased the severity of premalignant lesions over 10 years of follow-up, but the effects on gastric adenocarcinoma per se were not reported. ${ }^{15} \mathrm{An}$ other trial conducted in China demonstrated that eradication of $H$. pylori after endoscopic resection of early gastric cancer may inhibit the development of new metachronous carcinomas. However, this trial was not randomized and only followed patients up to 3 years. ${ }^{16}$ These data underscore the need for studies that can more closely define the effects of $H$. pylori eradication on the development of gastric cancer.

Rodent models have provided valuable insights into the host, bacterial, and environmental factors involved in gastric carcinogenesis. ${ }^{17}$ Long-term ( $>1$ year) $\mathrm{H}$. pylori infection of Mongolian gerbils can lead to gastric adenocarcinoma, without the coadministration of carcinogens, and gastric cancer in this model occurs in the distal stomach, as in humans. ${ }^{18-20}$ However, before 2005, the development of gastric cancer in gerbils had never been demonstrated outside east Asia, which limited the widespread use of this model. ${ }^{18-20}$ Another limitation of this system was the prolonged time course required for malignancy to develop, which precludes large-scale analyses that can evaluate the effects of pathogen, host, or intervention therapy, in the carcinogenic cascade.

We recently reported that an in vivo-adapted $H$. pylori strain (7.13) reproducibly induces gastric cancer in Mongolian gerbils, a process that did not occur when gerbils were infected with its progenitor human clinical strain B128. ${ }^{21}$ Following infection with strain 7.13 , gastric adenocarcinoma develops in approximately $60 \%$ of $H$. pylori-infected gerbils by 16-20 weeks post-inoculation, which is accompanied by gastric dysplasia. ${ }^{21}$ Gastric carcinoma also developed without the coadministration of additional carcinogens, ${ }^{21}$ which has frequently been required to induce malignancy in this model. ${ }^{22}$ Thus, rodent adaptation led to the identification of a $H$. pylori strain that rapidly and reproducibly induces gastric cancer in a highly penetrant fashion. As the effects of $H$. pylori eradication on development of gastric carcinoma remain unclear, we utilized this isolate as a prototype to define the role of antimicrobial therapy in a model of accelerated $H$. pylori-mediated carcinogenesis. The results indicate that treatment of $H$. pylori decreases the incidence and the severity of lesions with carcinogenic potential and pro-inflammatory cytokine expression, and that the effectiveness of eradication is dependent on the timing of intervention, providing a framework for understanding how malignancies arise within the context of H. pylori-induced inflammation.

\section{MATERIALS AND METHODS}

\section{Animals and Housing}

Outbred, male, specific pathogen-free Mongolian gerbils of approximately $50 \mathrm{~g}$ body weight, corresponding to an age of 4-8 weeks, were purchased from Harlan Sprague Dawley Inc. ${ }^{21}$ Five animals per cage were housed in the Animal Care Facilities of Vanderbilt University in a room with a 12 -h light:dark cycle at $21-22^{\circ} \mathrm{C}$ and were fed a standard commercial rodent chow (Rodent LabDiet 5001, TestDiet, Division of Land O'Lakes Purina Feed, LLC, Richmond, IN, USA). All experiments and procedures were approved by the Institutional Animal Care Committee of Vanderbilt University.

\section{H. pylori Challenge and Antibiotic Therapy}

The gerbil-passaged H. pylori strain 7.13 isolate used for these experiments was originally isolated from a gastric ulcer patient. ${ }^{21} H$. pylori strains for each challenge were grown from freezer stock on trypticase soy agar (TSA) plates containing $5 \%$ sheep blood, at $37^{\circ} \mathrm{C}$ with $5 \% \mathrm{CO}_{2}$, and then inoculated into Brucella broth containing 5\% heat-inactivated fetal bovine serum and $10 \mathrm{mg} / \mathrm{ml}$ vancomycin. ${ }^{21}$ After $12 \mathrm{~h}$, cells were harvested by centrifugation. Gerbils were fasted overnight and orogastrically inoculated two times (days 0 and 3) with $1.0 \mathrm{ml}$ of an $H$. pylori cell suspension of $5 \times 10^{9} \mathrm{CFU} / \mathrm{ml}$ in sterile Brucella broth through a feeding needle as described previously. ${ }^{21}$ Control animals were inoculated with identical volumes of sterile Brucella broth.

Antibiotic therapy was delivered at 4 (Protocol a) or 8 (Protocol b) weeks after $H$. pylori challenge (Figure 1) and consisted of lansoprazole $(1 \mathrm{mg} / \mathrm{kg}$ ) (TAP Pharmaceuticals Inc., Lake Forest, IL, USA), amoxicillin ( $15 \mathrm{mg} / \mathrm{kg}$ ) (Ranbaxy Pharmaceuticals, Jacksonville, FL, USA), and clarithromycin $(30 \mathrm{mg} / \mathrm{kg}$ ) (Abbott Laboratories, North Chicago, IL, USA) dissolved in a total volume of $500 \mu \mathrm{l}$ sterile water and was administrated via oral gavage daily for 14 days. These time points were selected based on our previous data indicating

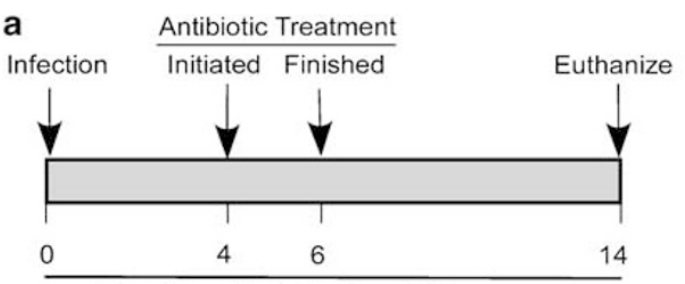

Duration of Infection (weeks)

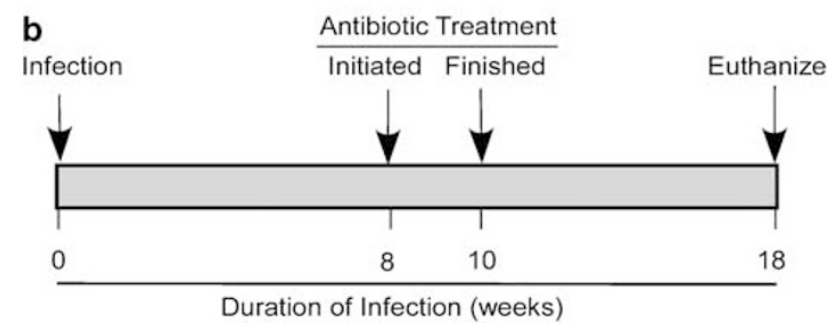

Figure 1 Eradication protocol. Mongolian gerbils were infected with H. pylori strain 7.13 for 4 (a) or 8 (b) weeks and then received either eradication or sham therapy for 2 weeks (arrows). Gerbils were then euthanized 8 weeks after completion of therapy. 
that the incidence of gastric carcinoma increased sharply between 4 and 8 weeks of infection with this prototype H. pylori strain. ${ }^{21}$ This regimen was selected based on previous studies demonstrating the efficacy of this therapy for H. pylori eradication in infected gerbils. ${ }^{22,23}$ Sham therapy consisted of $500 \mu$ l sterile water delivered daily for 14 days. Infected and uninfected gerbils, treated with antibiotics or water, were euthanized 8 weeks post-treatment (Figure 1).

\section{Histological Examination}

Upon euthanization, gerbil stomachs were divided longitudinally into two halves. For one-half of the stomach, linear strips extending from the squamocolumnar junction through proximal duodenum were fixed in $10 \%$ neutral-buffered formalin, paraffin-embedded, stained with hematoxylin and eosin (H\&E), and indices of inflammation and injury were scored by a single pathologist $(\mathrm{EH})$ blinded to treatment groups. ${ }^{21}$ The following parameters were graded on a $0-3$ scale in the gastric antrum and corpus: acute inflammation, chronic inflammation (mononuclear cell infiltration independent of lymphoid follicles), cryptitis, and colonization density of $H$. pylori in the gastric glands and foveolae or adjacent to the epithelium. ${ }^{21}$ Lymphoid follicle formation was graded on a $0-2$ scale as described previously. ${ }^{21}$ Dysplastic mucosa was graded as 0 (absent), 1 (focal), or 2 (extensive) and consisted of irregular, angulated, and occasionally cystically dilated glands with enlarged, overlapping hyperchromatic nuclei. Carcinoma was defined as irregular, angulated, cystically dilated glands with occasional cribriform architecture in the submucosa and muscularis propria, spreading laterally to the surface mucosal component. $^{21,24}$

The other half of the stomach was divided into two parts: one half was transferred to a sterile tube and stored at $-80^{\circ} \mathrm{C}$ for RNA extraction, while the other half was transferred to $1.0 \mathrm{ml}$ of sterile phosphate-buffered saline (PBS, $\mathrm{pH} 7.4$ ), homogenized, and plated onto selective TSA $/ 5 \%$ sheep blood plates containing vancomycin $(20 \mu \mathrm{g} / \mathrm{ml})$, bacitracin $(200 \mu \mathrm{g} /$ ml) (Calbiochem, San Diego, CA, USA), nalidixic acid $(10 \mu \mathrm{g} / \mathrm{ml})$, and amphotericin B $(2 \mu \mathrm{g} / \mathrm{ml})$ (Sigma Chemical Co, St Louis, MO, USA) and grown for 5-7 days as described previously. ${ }^{21} H$. pylori was identified by morphology and urease and oxidase activity. To assess antibiotic resistance, the minimal inhibitory concentrations for amoxicillin, clarithromycin, and metronidazole were determined using the Epsilometer test (E-test; AB Biodisk, Piscataway, NJ, USA) on TSA-5\% sheep blood plates (BBL, Cockeysville, MD, USA) as described previously. ${ }^{25}$ Briefly, inocula from each $H$. pylori strain were harvested from agar plates after $48 \mathrm{~h}$ of incubation and bacteria were suspended in sterile saline to a turbidity that was equivalent to a Mcfarland standard of 1. Bacteria were then swabbed onto agar plates and allowed to dry before E-test strips were applied. Plates with strips containing metronidazole were incubated under strict anaerobic conditions for $24 \mathrm{~h}$ and then switched to microaerobic conditions for $24 \mathrm{~h}$. Plates with strips containing amoxicillin or clarithromycin were incubated in microaerobic conditions for $72 \mathrm{~h}$. Thresholds for resistance were defined according to the manufacturers' instructions.

\section{RNA Extraction and RT-PCR}

Gastric tissue from uninfected and infected gerbils was placed into sterile vials (Nunc, Roskilde, Denmark) and stored at $-80^{\circ} \mathrm{C}$ until used for RNA preparation for RT-PCR. Total RNA was isolated using TRIzol reagent (Invitrogen, Carlsbad, CA, USA) according to the manufacturer's instructions. RNA was dissolved in $100 \mu$ l diethyl pyrocarbonate-treated distilled water and purified using RNeasy (Qiagen, Valencia, CA, USA). A $1 \mu$ g portion of RNA was then used for first strand cDNA synthesis using Taqman Reverse Transcription reagents (Applied Biosystems, Foster City, CA, USA).

PCR primers for use with random hexamer-primed cDNA as template were designed based on published gerbil sequences of IFN- $\gamma$ and $\beta$-actin. ${ }^{26}$ Primer sequences were as follows: IFN- $\gamma$, sense ( $5^{\prime}$ ccatgaacgctacacactgcatc), antisense ( $5^{\prime}$ gaagtagaaagagacaatctgg); $\beta$-actin, sense ( $5^{\prime}$ gcaccacaccttctac aatgag), antisense ( $5^{\prime}$ atagcacagcctggatagcaac). PCR was carried out in a total volume of $50 \mu \mathrm{l}$ containing PCR buffer $(50 \mathrm{mM} \mathrm{KCl}, 10 \mathrm{mM}$ Tris/HCl, $\mathrm{pH} 8.3), 1.5 \mathrm{mM} \mathrm{MgCl}_{2}$, $200 \mu \mathrm{M}$ (each) deoxynucleotides, $2 \mathrm{U}$ of AmpliTaq polymerase (Perkin-Elmer Cetus), and $1 \mu \mathrm{l}$ of cDNA as template. All primers were used at a final concentration of $0.5 \mu \mathrm{M}$. Each reaction mixture was amplified for 35 cycles as follows: $1 \mathrm{~min}$ at $94^{\circ} \mathrm{C}, 45 \mathrm{~s}$ annealing at $60^{\circ} \mathrm{C}(\mathrm{IFN}-\gamma)$ or $55^{\circ} \mathrm{C}$ ( $\beta$-actin), and $1 \mathrm{~min}$ extension at $72^{\circ} \mathrm{C}$. After the 35 th cycle, extension was continued for another $7 \mathrm{~min}$. Agarose gel electrophoresis with ethidium bromide staining was performed on an aliquot from each PCR. To avoid contamination, PCR reactions were prepared in a dedicated area used only for PCR and PCR products were opened in a laminar flow hood separated from the PCR preparation area. Negative and positive control amplifications were performed in every experiment.

\section{Statistical Analysis}

Gerbils were considered to be successfully infected if results from histological examination of tissue and culture were concordantly positive. The Mann-Whitney $U$-test was used for statistical analyses of intergroup comparisons. A log-rank test was used to determine the differences of disease outcome between groups. Significance was defined as $P<0.05$.

\section{RESULTS}

\section{Colonization Efficiency of $\boldsymbol{H}$. pylori}

Success of $H$. pylori challenge was determined by quantitative culture and histological assessment. No animals challenged with Brucella broth alone had detectable evidence of $H$. pylori. Twenty-three gerbils were challenged with $H$. pylori and did not receive antibiotics (Table 1); 18 (78\%) of these 23 were successfully infected as determined by culture 
Table 1 Colonization efficiency and efficacy of antimicrobial therapy directed against $\boldsymbol{H}$. pylori

\begin{tabular}{lccccc}
\hline $\begin{array}{l}\text { Duration of infection prior } \\
\text { to therapy }\end{array}$ & \multicolumn{2}{c}{4 weeks } & & \multicolumn{2}{c}{8 weeks } \\
\cline { 2 - 3 } \cline { 5 - 6 } \cline { 5 - 6 } & Antibiotics & Water & & Antibiotics & Water \\
Gerbils challenged & 20 & 9 & & 15 & 14 \\
Culture positive & 0 & 8 & & 1 & 10 \\
Culture negative & 20 & 1 & & 14 & 4 \\
\hline
\end{tabular}

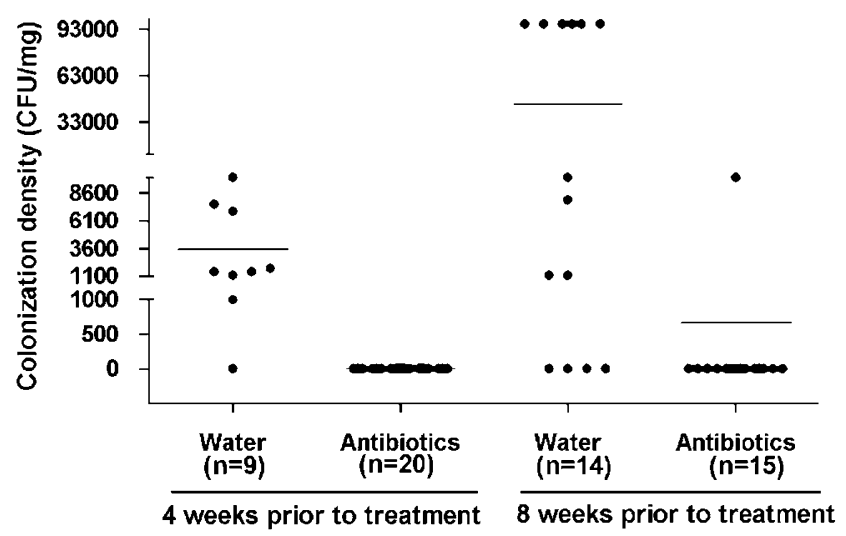

Figure 2 Colonization density within antral mucosa of Mongolian gerbils infected with H. pylori for 4 or 8 weeks prior to treatment with water or antibiotics. Colonization density was determined by quantitative culture and data are presented as scatter plots with mean values of colony-forming units (CFU) per milligram of tissue.

and histology. Colonization density was determined by quantitative culture (Figure 2).

Inflammation and Carcinogenesis Induced by $\boldsymbol{H}$. pylori Next, we examined the parameters of inflammation and carcinogenesis in gerbils infected with $H$. pylori strain 7.13 alone (no antibiotic treatment group). There was no evidence of gastric inflammation or injury in uninfected control animals that were followed out to 14 (Figure $3 \mathrm{a}$ ) or 18 (Figure 4a) weeks post-challenge. As expected, all gerbils infected with $H$. pylori developed significantly higher inflammatory scores compared with uninfected controls $(P<0.05)$. Chronic active gastritis developed in the majority of gerbils that were successfully infected with $H$. pylori strain 7.13, which was characterized by acute inflammation frequently accompanied by an abundant lymphoplasmacytic infiltrate involving antral mucosa and submucosa, with lymphoid follicle formation (Figures $3 \mathrm{c}$ and $4 \mathrm{c}$ ). There were no significant correlations between colonization density (Figure 2) and the severity of inflammation in gerbils infected with $H$. pylori.

Gastric dysplasia and adenocarcinoma developed in the gastric antra of 67 and 33\%, respectively, of gerbils infected with strain 7.13 for 14 weeks (Figure 5, left panel). By 18 weeks of infection, 64 and $50 \%$ of $H$. pylori-infected gerbils that did not receive antibiotics developed gastric antral dysplasia or cancer (Figure 5, right panel). Antral tumors were characterized by irregular, angulated, dilated glands invading into the submucosa and muscularis propria, and were accompanied by surface antral dysplasia and marked chronic active gastritis (Figures $3 \mathrm{~d}$ and $4 \mathrm{~d}$ ). These results indicate that infection with $H$. pylori strain 7.13 rapidly and reproducibly leads to the development of gastric dysplasia and adenocarcinoma, which provided a framework to assess the effects of $H$. pylori eradication in this model of gastric cancer.

Efficacy of Antibiotic Therapy for Eradication of $\boldsymbol{H}$. pylori Having established that our prototype strain 7.13 could induce the pre-malignant and malignant lesions of interest, we next investigated the effects of treatment on these events. Thirty-five gerbils were challenged with $H$. pylori strain 7.13 and subsequently received antibiotic therapy at either 4 ( $n=20$ gerbils) or 8 ( $n=15$ gerbils) weeks post-inoculation (Table 1). Of the $35 \mathrm{H}$. pylori-infected gerbils that received antibiotics, $34(97 \%)$ were culture and histology negative when killed, indicating that this antibiotic regimen was effective for eradicating $H$. pylori.

The single gerbil that received antimicrobial therapy but remained persistently colonized was infected with strain 7.13 for 8 weeks prior to receiving antibiotics. To determine whether acquired antibiotic resistance represented a potential explanation for eradication failure, we tested the recovered isolate as well as the input strain 7.13 for susceptibility to metronidazole, amoxicillin, and clarithromycin. Both isolates were susceptible to clarithromycin and amoxicillin but resistant to metronidazole. There were no differences in levels of sensitivity to any of the antibiotics tested between the input isolate and the recovered strain, indicating that acquired antibiotic resistance was not responsible for failure of antimicrobial therapy in this case.

\section{Effect Of $\boldsymbol{H}$. pylori Eradication on Parameters of Gastric Inflammation}

Having established the efficacy of this antibiotic regimen for elimination of $H$. pylori, we next quantified the effects of microbial eradication on the development of pre-malignant and malignant lesions in this model. Compared with gerbils that remained persistently infected (no antibiotic treatment group), $H$. pylori-challenged gerbils that subsequently received antibiotics 4 or 8 weeks later had significantly decreased gastric antral acute (mean + s.e.m. $0.0+0.0$ $v s 2.1+0.3$ at 4 weeks post-treatment, Figure $6 \mathrm{a} ; 0.3+0.18 v s$ $1.9+0.3$ at 8 weeks post-treatment, Figure $6 \mathrm{~b}$; antibiotictreated $v s$ placebo, respectively) and chronic (mean + s.e.m. $0.1+0.05$ vs $2.4+0.3$ at 4 weeks post-treatment, Figure 6 c; $1.0+0.3$ vs $2.1+0.4$ at 8 weeks post-treatment, Figure $6 \mathrm{~d}$; antibiotic-treated $v s$ placebo, respectively) inflammatory scores. In the gastric corpus, the severity of chronic 

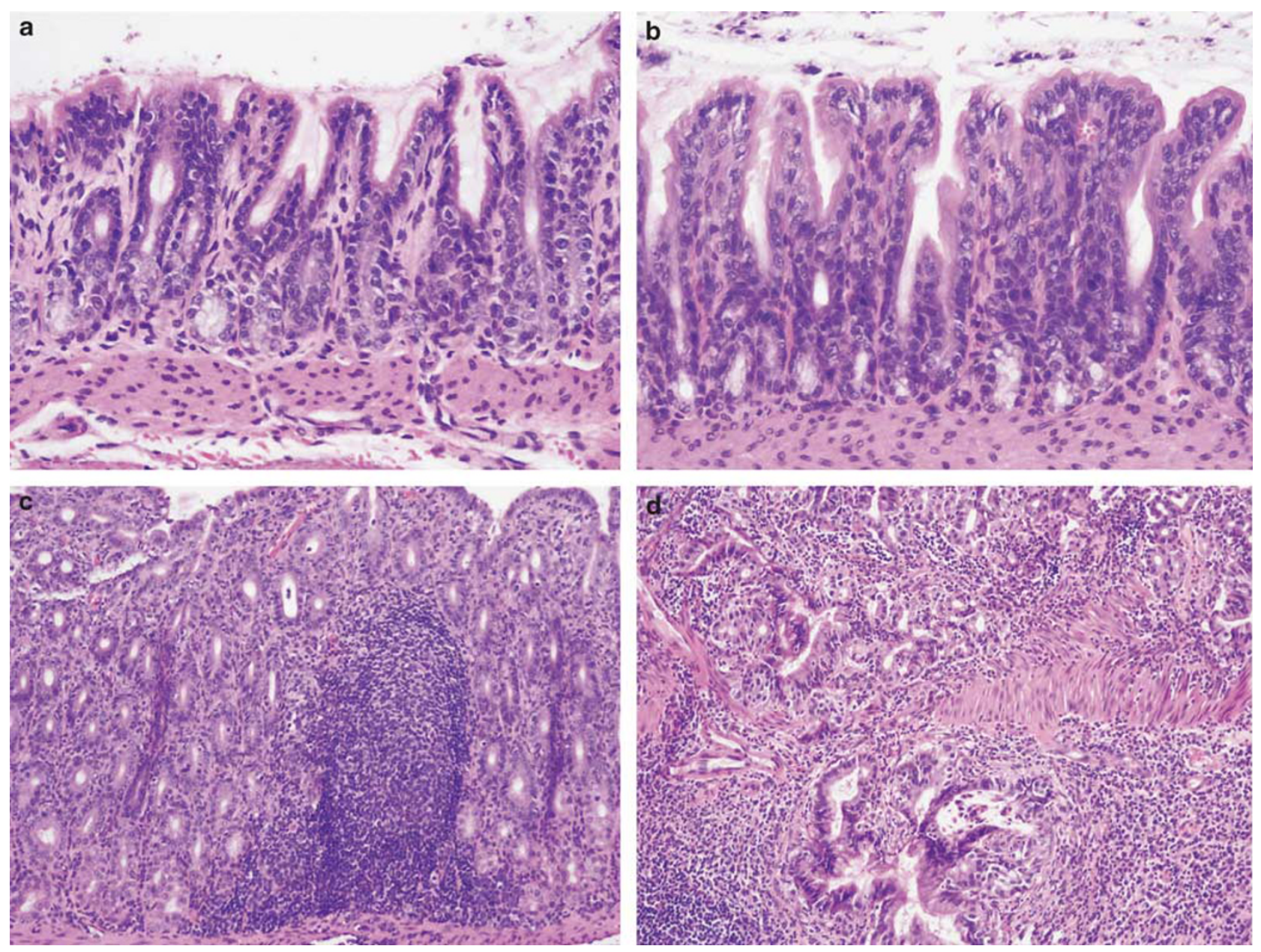

Figure 3 Gastric inflammation and injury in gerbils that are uninfected or infected with $H$. pylori for 4 weeks and subsequently treated with or without antibiotics. Representative H\&E stains are shown for (a) uninfected, (b) H. pylori-infected and subsequently treated with antibiotics, and (c and d) $H$. pyloriinfected and subsequently treated with sham therapy. No significant pathological abnormalities were present in gerbils challenged with broth alone (a) or with H. pylori followed by antimicrobial therapy (b). Lymphoid follicles (c) and gastric carcinomas (d) frequently arose within a background of inflammation. Gastric carcinomas were characterized by marked cellular pleomorphism, cellular atypia, and euchromatic nuclei that exhibited bizarre morphologic features. Magnification, $\times 10$.

inflammation at both time points and acute inflammation at the 8-week time point was also significantly decreased in gerbils that received antibiotic therapy as compared with the no therapy group (Figures $6 \mathrm{~b}-\mathrm{d}$ ).

The timing of intervention also influenced the magnitude of attenuation. Among gerbils that received antibiotics 4 weeks post-challenge, only one animal had evidence of chronic inflammation, which was classified as mild (Figure 6c). Delaying therapy until 8 weeks post-challenge resulted in reduced efficacy for attenuation of chronic inflammation, as many of the antibiotic-treated gerbils still had evidence of gastritis, although inflammatory scores were significantly lower compared with gerbils that were persistently infected with $H$. pylori (Figure 6d). In contrast, the severity of acute inflammation was efficiently suppressed at both time points by anti- $H$. pylori therapy (Figure $6 \mathrm{a}$ and $\mathrm{b}$ ).

\section{Effect of $\boldsymbol{H}$. pylori Eradication on Gastric Mucosal Pro-Inflammatory Cytokine Expression}

The inflammation and injury response to $H$. pylori is mediated in part by Th1-type cytokines, such as IFN- $\gamma .^{11,12}$ To determine whether this response may underpin the timedependent effect of antibiotics on gastric inflammation, we examined expression of IFN- $\gamma$ and, as a control, $\beta$-actin within gerbil gastric antral tissue. For this analysis, we excluded gerbils challenged with $H$. pylori that were not successfully colonized ( $n=5$, Table 1$)$, and gerbils for which antibiotic therapy did not result in eradication $(n=1)$. There was no detectable IFN- $\gamma$ expression in any of the uninfected gerbils. Compared with gerbils that remained persistently infected (no antibiotic treatment group, $n=8$ ), significantly $(P=0.0017)$ fewer $H$. pylori-challenged gerbils that received antibiotics 4 weeks later $(n=20)$ had detectable IFN- $\gamma$ as 

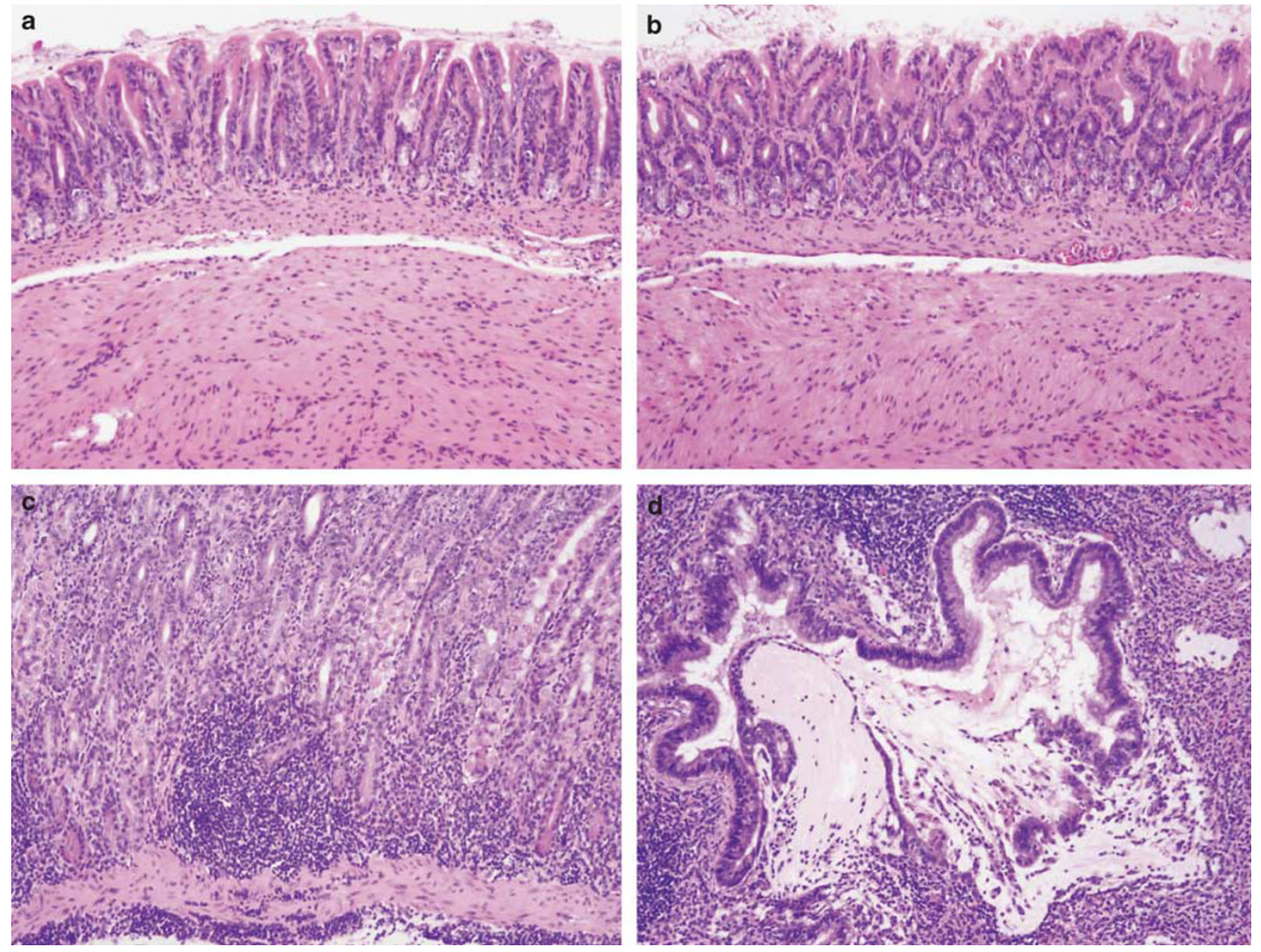

Figure 4 Gastric inflammation and injury in gerbils that are uninfected or infected with $\mathrm{H}$. pylori for 8 weeks and subsequently treated with or without antibiotics. Representative H\&E stains are shown for (a) uninfected, (b) H. pylori-infected and subsequently treated with antibiotics, and (c and d) H. pylori-infected and subsequently treated with sham therapy. No significant pathologic abnormalities were present in gerbils challenged with broth alone (a) and inflammation was decreased, but not abolished, in gerbils infected with $\mathrm{H}$. pylori, followed by antimicrobial therapy (b). Lymphoid follicles (c) and gastric carcinomas (d) frequently arose within a background of inflammation. Magnification $\times 10$.

assessed by RT-PCR (Figure 7). In contrast, the frequency of detection of IFN- $\gamma$ expression did not differ $(P=0.398)$ between antibiotic-treated $(n=14)$ and control-treated $(n=10)$ gerbils, when the therapy was delayed until 8 weeks post-challenge (Figure 7). These data indicate that the pattern of Th1 cytokine expression mirrors the pattern of chronic inflammation in gerbils infected for different periods of time before initiation of antibiotics.

\section{Effect of Antibiotic Therapy on Gastric Dysplasia and Carcinoma}

To determine whether antibiotic suppression of $H$. pyloriinduced inflammation affected lesions with pre-malignant potential, we focused on gastric dysplasia as well as carcinoma in $H$. pylori-challenged gerbils. None of the $H$. pylori-challenged gerbils that received antibiotics 4 weeks post-inoculation developed gastric dysplasia or adenocarcinoma (Figure 5, left panel). The incidence of dysplasia (13\%) and carcinoma (7\%) was also significantly reduced, but not completely abolished, in gerbils treated with antibiotics 8 weeks post- $H$. pylori challenge (Figure 5, right panel). Collectively, these results demonstrate that treatment of $H$. pylori in this model of gastric carcinogenesis decreases the incidence and the severity of lesions with carcinogenic potential, but that the effectiveness of eradication is dependent upon the timing of intervention.

\section{DISCUSSION}

A biological consequence of long-term $H$. pylori colonization is an increased risk of gastric adenocarcinoma. By capitalizing on a rodent model that recapitulates human disease and utilizing an $H$. pylori strain that rapidly induces gastric cancer, our current results demonstrate that treatment of $H$. pylori can prevent the subsequent development of gastric carcinoma. However, the effectiveness of eradication is dependent upon the timing of intervention. 
Studies that have focused on the consequences of $H$. pylori eradication in human populations have yielded mixed results. Wong et $\mathrm{al}^{13}$ investigated the effects of anti-H. pylori therapy

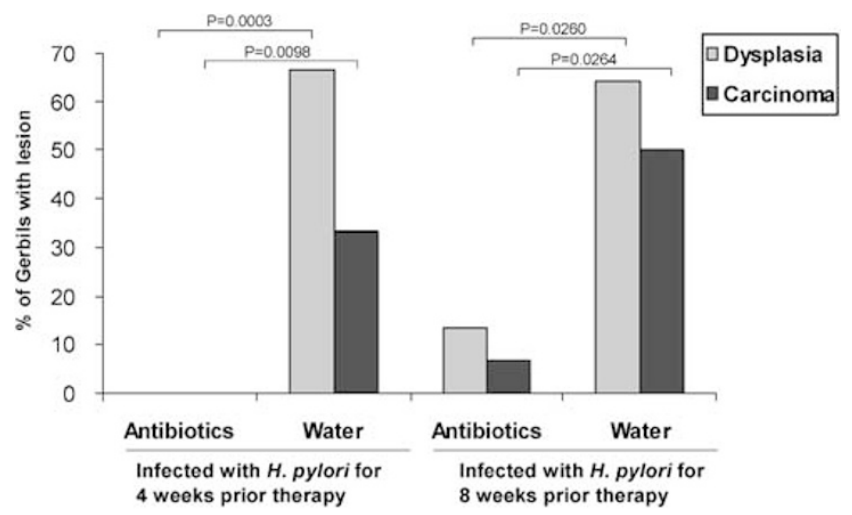

Figure $\mathbf{5}$ The development of gastric dysplasia and adenocarcinoma is attenuated by antimicrobial therapy directed against $H$. pylori. Chronology of gastric dysplasia (light shaded columns) and adenocarcinoma (dark shaded columns) in Mongolian gerbils challenged with H. pylori strain 7.13 for 4 or 8 weeks and then treated with or without antibiotic therapy. Results are expressed as a percentage of gerbils with dysplasia or adenocarcinoma infected for 4 or 8 weeks prior to initiation of antibiotics or sham therapy. No dysplasia or adenocarcinoma developed in gerbils infected with strain 7.13 for 4 weeks prior to antibiotic treatment. The development of gastric dysplasia and carcinoma was significantly attenuated, but not eliminated, in gerbils infected for 8 weeks prior to antibiotic therapy.
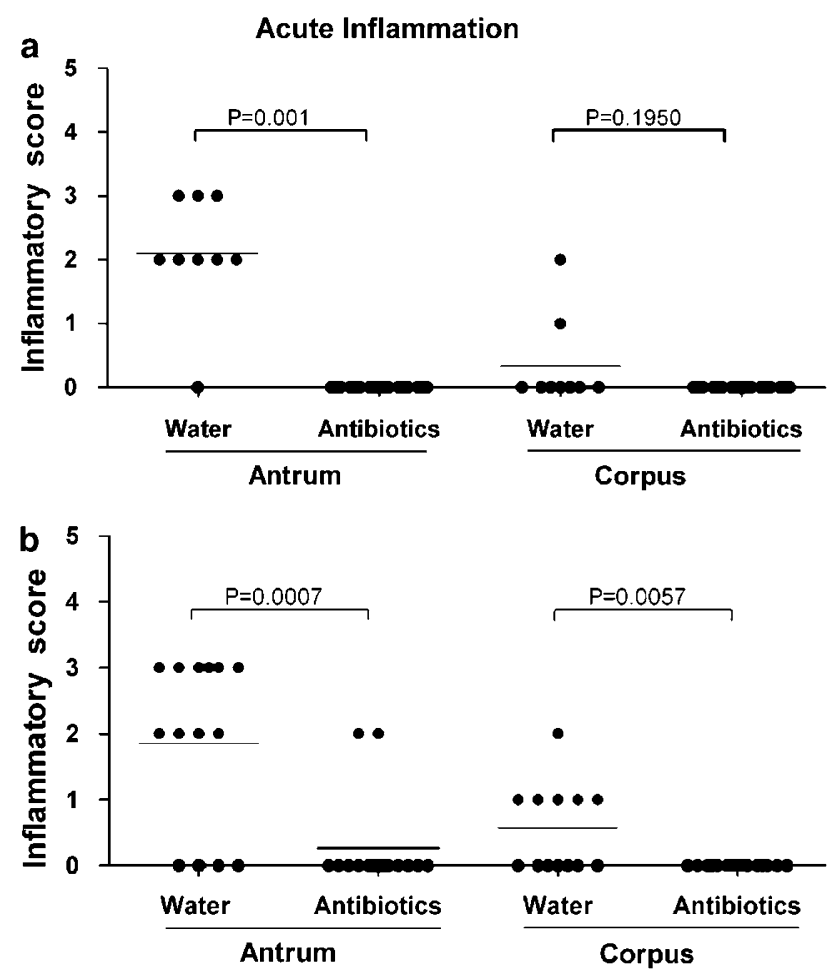

in a population of infected subjects residing in China. Among $>800$ persons who received antibiotics, 7 new cases of gastric cancer developed over a mean follow-up period of 7.5 years, compared with 11 new cases that developed in placebotreated persons and who, therefore, remained persistently infected. ${ }^{13}$ Although these differences were not statistically significant, a secondary analysis revealed that treatment of $H$. pylori was effective in preventing gastric cancer in persons who lacked pre-malignant lesions (eg, gastric atrophy, intestinal metaplasia, and dysplasia). You et al $^{14}$ reported that the combined incidence of atrophy, intestinal metaplasia, dysplasia, and cancer were reduced following treatment directed against $H$. pylori, but when the incidence of gastric cancer was analyzed separately, there was no significant benefit for eradication therapy.

A limitation of these studies was that the mean follow-up period following intervention ranged from 4 to 8 years, and gastric cancer usually develops only after decades of $H$. pyloriinduced injury. This has prompted additional trials to focus specifically on gastric cancer precursor lesions. In support of this, a recently completed chemoprevention trial in Colombia demonstrated that earlier interventions against $H$. pylori lead to more effective reductions in the severity of pre-malignant lesions compared with later interventions. ${ }^{15}$ Thus, the prevention process, similar to gastric carcinogenesis itself, follows an exponential curve in which the initial years of
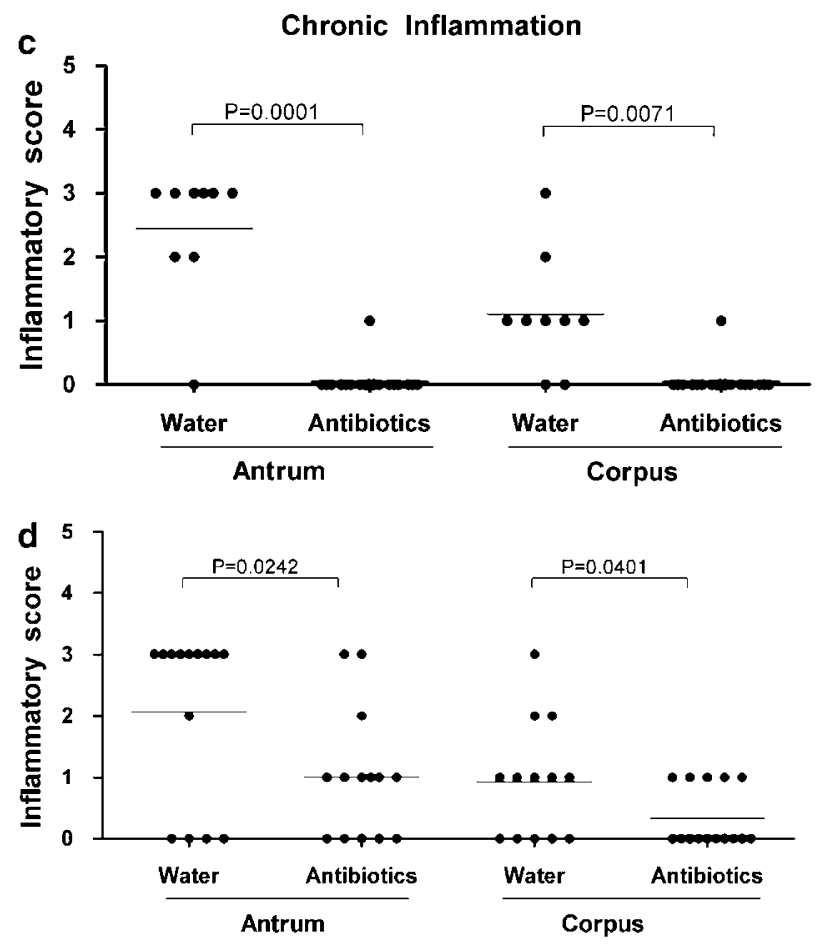

Figure 6 Severity of inflammation within the gastric antra and corpus of Mongolian gerbils inoculated with $H$. pylori and then treated with or without antibiotic therapy. Comparison of acute (a and $\mathbf{b})$ and chronic (c and d) gastric inflammation in Mongolian gerbils infected with $H$. pylori strain 7.13 for 4 (a and $\mathbf{c}$ ) or 8 (b and d) weeks prior to initiation of antibiotics or water. Inflammation was significantly attenuated in gerbils infected with $H$. pylori strain 7.13 that received antibiotics compared with infected gerbils treated with sham therapy for all time points, except for acute corpus inflammation at 4 weeks. Inflammation was determined by histological examination, as described in the text, and data are presented as scatter-plots with mean values. 

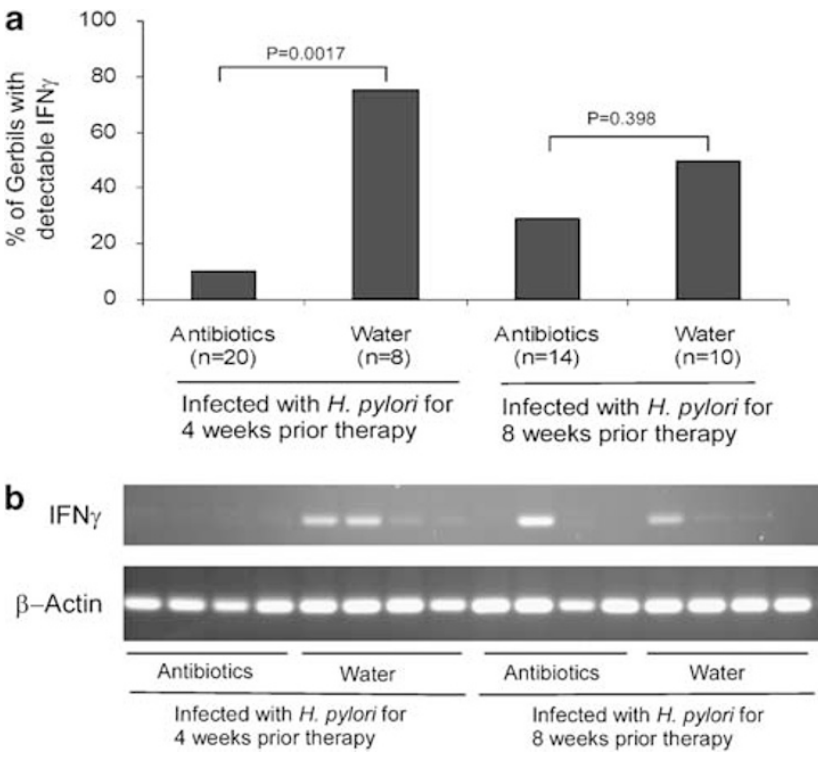

Figure 7 Detection of pro-inflammatory gene expression within the gastric antra of Mongolian gerbils inoculated with $H$. pylori and then treated with or without antibiotic therapy. (a) Comparison of detectable IFN- $\gamma$ expression in Mongolian gerbils infected with H. pylori strain 7.13 for 4 or 8 weeks prior to initiation of antibiotics or water. Results are expressed as a percentage of gerbils with detectable IFN- $\gamma$ expression infected for 4 or 8 weeks prior to initiation of antibiotics or sham therapy. IFN- $\gamma$ was detected significantly $(P=0.0017)$ less frequently in gerbils infected with $H$. pylori strain 7.13 for 4 weeks that received antibiotics compared with infected gerbils treated with sham therapy. However, these differences resolved in gerbils infected for 8 weeks prior to initiation of therapy. Gene expression was determined by RT-PCR, as described in the text. (b) Representative RTPCR amplicons from gerbils infected with strain 7.13 for 4 or 8 weeks prior to initiation of antibiotics or water. $\beta$-actin was used as a normalization control for RNA expression.

exposure (or lack thereof) have minimal measurable effects on markers of progression, but this is followed by greater effects in subsequent years. ${ }^{27}$ However, this study did not investigate the effects of $H$. pylori eradication on the endpoint outcome of gastric cancer.

Animal models are useful in that they represent tractable systems that permit insights into the effects of host, pathogen, and environmental factors on gastric carcinogenesis. ${ }^{17} \mathrm{Cao}$ et $\mathrm{al}^{23}$ reported that antimicrobial therapy induces apoptosis and inhibits proliferation in $H$. pylori-infected gerbil gastric mucosa. However, the effect of $H$. pylori eradication on pre-malignant (eg, dysplasia) and malignant histological lesions was not examined. Treatment of $H$. pylori in gerbils has been reported to decrease tumor formation; however, this required coadministration of both $H$. pylori and $N$-methyl- $N$-nitrosourea to induce tumors, ${ }^{22}$ which does not completely reflect $H$. pylori-induced cancer in humans. An advantage of our current model is that gastric carcinoma develops following infection with $H$. pylori per se, and occurs within a time frame sufficient to rigorously evaluate endpoints following intervention.
Transgenic mice that overexpress gastrin (INS-GAS mice) spontaneously develop gastric cancer, but this requires the virtual lifetime of the animal. ${ }^{28}$ Concomitant infection with $H$. pylori or a related Helicobacter species, $H$. felis, accelerates this process, suggesting that persistently elevated gastrin levels synergize with Helicobacter to augment cancer progression. ${ }^{28-30} \mathrm{H}$. felis has also been used to induce gastric adenocarcinoma in wild-type C57/BL6 mice, with virtually all colonized mice developing carcinoma by 15 months of infection. ${ }^{31}$ Cai $e t ~ a l^{31}$ utilized this model to demonstrate that targeted antimicrobial therapy can reverse inflammation, metaplasia, and dysplasia in $\mathrm{H}$. felis-infected C57/Bl6 mice if delivered within 6 months of infection. Eradication therapy given at later time points when more severe grades of metaplasia and dysplasia were present resulted in partial reversion of these lesions, and the incidence of cancer-related gastric outlet obstruction and death was also decreased. ${ }^{31}$ Our findings are consistent with this model, as we demonstrated that the timing of intervention influences the magnitude of suppression of pro-inflammatory cytokine expression, inflammation, pre-malignant, and neoplastic lesions. In contrast to infection with $\mathrm{H}$. felis, however, our model provides additional advantages for future mechanistic studies. Many H. pylori virulence components (eg, cag island) are not present within the genome of $H$. felis. Complete genome sequences from three different $H$. pylori strains are also currently available, ${ }^{32-34}$ which will facilitate studies that can more precisely delineate the relationship between genetic composition and phenotypes related to cancer.

Among the population of gerbils that received antibiotics 4 weeks post $H$. pylori challenge, significantly less animals had detectable levels of IFN- $\gamma$ expression, and no cases of dysplasia or carcinoma were present. Delaying therapy until 8 weeks resulted in a reduced efficacy for attenuating proinflammatory gene expression, inflammation, dysplasia, or carcinoma, suggesting that cytokines may have influenced the carcinogenic phenotype in this model. Indeed, there are several potential mechanisms through which Th1-type cytokines may augment the risk of $H$. pylori-induced gastric cancer. Intervention studies in mice have demonstrated that modulation of a pro-inflammatory Th1 response towards a Th2 response can prevent the development of the premalignant lesion gastric atrophy. ${ }^{35}$ Increased mucosal levels of IFN- $\gamma$ are associated with STAT3 activation, which likely mediates gastric cancer that develops in mice harboring a mutated IL-6 receptor. $^{36,37}$ Finally, Th1-polarized gastric responses are linked to reduced mucosal somatostatin and elevated plasma gastrin levels in $H$. pylori-infected patients, ${ }^{10}$ and increased gastrin levels are significantly associated with increased gastric epithelial cell proliferation. ${ }^{38}$

Establishment of H. pylori as a risk factor for cancer of the stomach has permitted an approach to identify persons at increased risk; however, infection with this organism is extremely common and most colonized persons never develop cancer. Analytical tools are now available, including 
genome sequences ( $H$. pylori and human), measurable phenotypes, and practical animal models, to identify high-risk subpopulations, allowing treatments to be designed and focused on individuals who are most likely to derive benefit from $H$. pylori eradication. Our current findings represent an important step in this process as we have demonstrated that treatment of $H$. pylori decreases the incidence and the severity of pro-inflammatory cytokine expression, as well as premalignant and malignant lesions; however, the effectiveness of eradication is dependent upon the timing of intervention. It is important to gain more insight into the pathogenesis of $H$. pylori-induced gastric adenocarcinoma, not only to develop more effective treatments for this cancer, but also because it might serve as a paradigm for the role of chronic inflammation in the genesis of other malignancies.

\section{ACKNOWLEDGEMENT}

This work was supported in part by National Institutes of Health Grants DK-73902, DK-58587, and CA-77955 (to RMP).

1. Peek Jr RM, Blaser MJ. Helicobacter pylori and gastrointestinal tract adenocarcinomas. Nat Rev Cancer 2002;2:28-37.

2. Correa P. Is gastric cancer preventable? Gut 2004;53:1217-1219.

3. Blanchard TG, Drakes ML, Czinn SJ. Helicobacter infection: pathogenesis. Curr Opin Gastroenterol 2004;20:10-15.

4. Houghton J, Wang TC. Helicobacter pylori and gastric cancer: a new paradigm for inflammation-associated epithelial cancers. Gastroenterology 2005;128:1567-1578.

5. Moss SF, Blaser MJ. Mechanisms of disease: inflammation and the origins of cancer. Nat Clin Pract Oncol 2005;2:90-97.

6. Ernst PB, Peura DA, Crowe SE. The translation of Helicobacter pylori basic research to patient care. Gastroenterology 2006;130:188-206.

7. Correa P. Helicobacter pylori and gastric cancer: state of the art. Cancer Epidemiol Biomarkers Prev 1996;5:477-481.

8. Uemura N, Okamoto S, Yamamoto S, et al. Helicobacter pylori infection and the development of gastric cancer. N Engl J Med 2001;345: 784-789.

9. Moss SF, Sood S. Helicobacter pylori. Curr Opin Infect Dis 2003;16: 445-451.

10. Peek Jr RM, Crabtree JE. Helicobacter infection and gastric neoplasia. J Pathol 2006;208:233-248.

11. Wilson KT, Crabtree JE. Immunology of Helicobacter pylori: insights into the failure of the immune response and perspectives on vaccine studies. Gastroenterology 2007;133:288-308.

12. Bamford KB, Fan $\mathrm{X}$, Crowe $\mathrm{SE}$, et al. Lymphocytes in the human gastric mucosa during Helicobacter pylori have a T helper cell 1 phenotype. Gastroenterology 1998;114:482-492.

13. Wong BC, Lam SK, Wong WM, et al. Helicobacter pylori eradication to prevent gastric cancer in a high-risk region of China: a randomized controlled trial. JAMA 2004;291:187-194.

14. You WC, Brown LM, Zhang L, et al. Randomized double-blind factorial trial of three treatments to reduce the prevalence of precancerous gastric lesions. J Natl Cancer Inst 2006;98:974-983.

15. Mera R, Fontham ET, Bravo LE, et al. Long term follow up of patients treated for Helicobacter pylori infection. Gut 2005;54:1536-1540.

16. Uemura $\mathrm{N}$, Mukai $\mathrm{T}$, Okamoto $\mathrm{S}$, et al. Effect of Helicobacter pylori eradication on subsequent development of cancer after endoscopic resection of early gastric cancer. Cancer Epidemiol Biomarkers Prev 1997;6:639-642.
17. Rogers $A B$, Fox JG. Inflammation and Cancer. I. Rodent models of infectious gastrointestinal and liver cancer. Am J Physiol Gastrointest Liver Physiol 2004;286:G361-G366.

18. Watanabe $T$, Tada $M$, Nagai $H$, et al. Helicobacter pylori infection induces gastric cancer in Mongolian gerbils. Gastroenterology 1998;115:642-648.

19. Honda S, Fujioka T, Tokieda M, et al. Development of Helicobacter pylori-induced gastric carcinoma in Mongolian gerbils. Cancer Res 1998;58:4255-4259.

20. Ogura K, Maeda S, Nakao M, et al. Virulence factors of Helicobacter pylori responsible for gastric diseases in Mongolian gerbil. J Exp Med 2000;192:1601-1610.

21. Franco AT, Israel DA, Washington MK, et al. Activation of $\beta$-catenin by carcinogenic Helicobacter pylori. Proc Natl Acad Sci USA 2005;102:10646-10651.

22. Nozaki K, Shimizu N, Ikehara $\mathrm{Y}$, et al. Effect of early eradication on Helicobacter pylori-related gastric carcinogenesis in Mongolian gerbils. Cancer Sci 2003;94:235-239.

23. Cao X, Tsukamoto T, Nozaki K, et al. Eradication of Helicobacter pylori induces apoptosis and inhibits proliferation of heterotopic proliferative glands in infected Mongolian gerbils. Cancer Sci 2004;95:872-877.

24. Boivin GP, Washington $\mathrm{K}$, Yang K, et al. Pathology of mouse models of intestinal cancer: consensus report and recommendations. Gastroenterology 2003;124:762-777.

25. Garza-Gonzalez E, Perez-Perez Gl, Alanis-Aguilar O, et al. Antibiotic susceptibility patterns of Helicobacter pylori strains isolated from northeastern Mexico. J Chemother 2002;14:342-345.

26. Crabtree JE, Court M, Aboshkiwa MA, et al. Gastric mucosal cytokine and epithelial cell responses to Helicobacter pylori infection in Mongolian gerbils. J Pathol 2004;202:197-207.

27. Peek Jr RM, Schneider BG, Correa P. Coming unglued. Clin Cancer Res 2006;12:2951-2954.

28. Wang TC, Dangler CA, Chen D, et al. Synergistic interaction between hypergastrinemia and Helicobacter infection in a mouse model of gastric cancer. Gastroenterology 2000;118:36-47.

29. Fox JG, Rogers AB, Ihrig M, et al. Helicobacter pylori-associated gastric cancer in INS-GAS mice is gender specific. Cancer Res 2003;63: 942-950.

30. Fox JG, Wang $T C$, Rogers $A B$, et al. Host and microbial constituents influence Helicobacter pylori-induced cancer in a murine model of hypergastrinemia. Gastroenterology 2003;124:1879-1890.

31. Cai X, Carlson J, Stoicov C, et al. Helicobacter felis eradication restores normal architecture and inhibits gastric cancer progression in C57BL/6 mice. Gastroenterology 2005;128:1937-1952.

32. Tomb JF, White $\mathrm{O}$, Kerlavage $\mathrm{AR}$, et al. The complete genome sequence of the gastric pathogen Helicobacter pylori. Nature 1997;388:539-547.

33. Alm RA, Ling LS, Moir DT, et al. Genomic-sequence comparison of two unrelated isolates of the human gastric pathogen Helicobacter pylori. Nature 1999;397:176-180.

34. Oh JD, Kling-Backhed $\mathrm{H}$, Giannakis $\mathrm{M}$, et al. The complete genome sequence of a chronic atrophic gastritis Helicobacter pylori strain: evolution during disease progression. Proc Natl Acad Sci USA 2006;103:9999-10004.

35. Fox JG, Beck P, Dangler CA, et al. Concurrent enteric helminth infection modulates inflammation and gastric immune responses and reduces Helicobacter-induced gastric atrophy. Nat Med 2000;6:536-542.

36. Zavros Y, Eaton KA, Kang W, et al. Chronic gastritis in the hypochlorhydric gastrin-deficient mouse progresses to adenocarcinoma. Oncogene 2005;24:2354-2366.

37. Judd LM, Alderman BM, Howlett M, et al. Gastric cancer development in mice lacking the SHP2 binding site on the IL- 6 family co-receptor gp 130. Gastroenterology 2004;126:196-207.

38. Peek RM, Wirth HP, Moss SF, et al. Helicobacter pylori alters gastric epithelial cell cycle events and gastrin secretion in Mongolian gerbils. Gastroenterology 2000;118:48-59. 